\title{
Validation of Submaximal Step Tests and the 6-Min Walk Test for Predicting Maximal Oxygen Consumption in Young and Healthy Participants
}

\author{
Sung Hyun Hong ${ }^{1}$, Hyuk In Yang $\left.{ }^{1}{ }^{(}\right)$, Dong-Il Kim ${ }^{2}{ }^{\mathbb{D}}$, Tomas I. Gonzales $\left.{ }^{3} \mathbb{(}\right)$, Soren Brage ${ }^{3}$ and \\ Justin Y. Jeon ${ }^{1, *(1)}$ \\ 1 Exercise Medicine Center for Diabetes and Cancer Patients, \\ ICONS and Department of Sport Industry Studies, Yonsei University, Seoul 03722, Korea; \\ shhong85@yonsei.ac.kr (S.H.H.); hyukin.yang@gmail.com (H.I.Y.) \\ 2 Department of Professional Therapy, Graduate School of Professional Therapy, Gachon University, \\ Seongnam, Gyeonggi-do 1342, Korea; dikim@gachon.ac.kr \\ 3 MRC Epidemiology Unit, Institute of Metabolic Science, Cambridge Biomedical Campus, \\ University of Cambridge, Cambridge CB2 1TN, UK; Tomas.Gonzales@mrc-epid.cam.ac.uk (T.I.G.); \\ soren.brage@mrc-epid.cam.ac.uk (S.B.) \\ * Correspondence: jjeon@yonsei.ac.kr; Tel.: +82-2-2123-6197
}

Received: 10 November 2019; Accepted: 29 November 2019; Published: 3 December 2019

\begin{abstract}
Background: This study aimed to test the validity of three different submaximal tests (i.e., 3-min step test with $20.3-\mathrm{cm}$ step box height $\left(3 \mathrm{MST}_{20}\right), 3-\mathrm{min}$ step test with 30-cm step box height (3MST 30$)$, and 6-min walk test (6MWT)) in estimating maximal oxygen consumption $\left(\mathrm{VO}_{2 \max }\right)$ in young and healthy individuals. Methods: The $3 \mathrm{MST}_{20}, 3 \mathrm{MST}_{30}, 6 \mathrm{MWT}$, as well as the cardiopulmonary exercise test (CPET) were performed in 73 participants (37 men and 36 women; mean age: $30.8 \pm 9.3$ years). All participants visited the clinic three times in a random order for anthropometric measurements, three submaximal tests, and the $\mathrm{VO}_{2 \max }$ test. Multiple linear regression analyses were conducted to construct the $\mathrm{VO}_{2 \max }$ prediction equations for each submaximal test. Results: The prediction equations developed based on multiple regression analyses for each submaximal tests were as follows: $3 \mathrm{MST}_{20}: \mathrm{VO}_{2 \max }=86.0-10.9 \times$ sex $($ male $=1$, female $=2)-0.4 \times$ age $-0.1 \times$ weight $-0.1 \times$ heart rate recovery at $30 \mathrm{~s}(\mathrm{HRR} 30 \mathrm{~s}) ; 3 \mathrm{MST}_{30}: \mathrm{VO}_{2 \max }=84.5-10.2 \times$ sex $($ male $=1$, female $=2)-0.4 \times$ age $-0.1 \times$ weight $-0.1 \times$ HRR30s; and $6 \mathrm{MWT}: \mathrm{VO}_{2 \max }=61.1-11.1 \times$ sex $($ male $=1$, female $=2)-0.4 \times$ age $-0.2 \times$ weight $-0.2 \times\left(\right.$ distance walked $\left.\cdot 10^{-1}\right)$. The estimated $\mathrm{VO}_{2 \max }$ values based on formulated equations were $37.0 \pm 7.9,37.3 \pm 7.6$, and $36.9 \pm 7.9 \mathrm{~mL} \cdot \mathrm{kg}^{-1} \cdot \mathrm{min}^{-1}$ derived from the $3 \mathrm{MST}_{20}, 3 \mathrm{MST}_{30}$, and $6 \mathrm{MWT}$, respectively. These estimated $\mathrm{VO}_{2 \max }$ values were not significantly different from the measured $\mathrm{VO}_{2 \max }$ value, $37.3 \mathrm{~mL} \cdot \mathrm{kg}^{-1} \cdot \mathrm{min}^{-1}$. The estimated $\mathrm{VO}_{2 \max }$ based on the $3 \mathrm{MST}_{20}, 3 \mathrm{MST}_{30}$, and $6 \mathrm{MWT}$ results explained $73.4 \%, 72.2 \%$, and $74.4 \%$ of the variances in the measured $\mathrm{VO}_{2 \max }(p<0.001)$, respectively. Conclusions: The $3 \mathrm{MST}_{20}, 3 \mathrm{MST}_{30}$, and $6 \mathrm{MWT}$ were valid in estimating $\mathrm{VO}_{2 \max }$ in relatively young and healthy Asian individuals.
\end{abstract}

Keywords: step test; 6-min walk test; validation; fitness

\section{Introduction}

Cardiopulmonary fitness has been used as an index of aerobic fitness for several decades [1]. However, direct measurement of cardiopulmonary fitness using gas analyzers can be costly and sometimes unsafe. Thus, indirect measurement has been considered using a step box [2], cycle ergometer [3], and treadmill [4,5]. Since the use of large equipment such as a cycle ergometer and 
treadmill could be difficult for field tests, various step tests have been developed and used as a surrogate method to estimate maximal oxygen consumption $\left(\mathrm{VO}_{2 \max }\right)[6]$.

There are two different types of step tests: Incremental multi-stage and single-stage step tests [4]. In incremental step tests, either the step box height or stepping rate is increased in an incremental manner [7], and various responses of participants during and after exercise are used to estimate $\mathrm{VO}_{2 \max }$ [8-10]. Although incremental step tests consider more variables such as heart rate responses, stepping rate or step height reached, and rate of perceived exertion (RPE) which is related to the participants' aerobic capacity, incremental step tests generally take a longer duration and elicit more physical stress [8-10]. Conversely, single-stage step tests mostly take a shorter duration and also only use heart rate recovery to estimate $\mathrm{VO}_{2 \max }$ [7]. Among single-stage step tests, the Tecumseh step test (3-min step test using 20.3-cm step box height $\left(3 \mathrm{MST}_{20}\right)$ ) [6] and the YMCA 3-min step test (3-min step test using $30-\mathrm{cm}$ step box height $\left(3 \mathrm{MST}_{30}\right)$ ) [2] have been used widely. Since the $3 \mathrm{MST}_{30}$ uses a higher step box height, local muscle fatigue and joint pain could be limiting factors in the elderly and participants with very low fitness levels or poor joint conditions $[2,11]$. Thus, it would be valuable to understand whether the $3 \mathrm{MST}_{20}$ is as good as the $3 \mathrm{MST}_{30}$ in estimating $\mathrm{VO}_{2 \max }$.

In addition to step tests, the 6-min walk test (6MWT), developed for patients with respiratory diseases, has been used extensively in the elderly [12] and clinical populations [13,14]. Since then, its validity has been tested in populations of various ages and health status; however, conflicting results have been reported [15-18]. Furthermore, whether the 6MWT is valid in estimating $\mathrm{VO}_{2 \max }$ in relatively young and healthy populations remains unclear. Although single-stage step tests and the 6MWT have been validated previously, they have not been validated in comparison with each other and against actual measured $\mathrm{VO}_{2 \max }$ in the same participants. Therefore, this study aimed to test the validity of three different submaximal tests (i.e., $3 \mathrm{MST}_{20}, 3 \mathrm{MST}_{30}$, and $6 \mathrm{MWT}$ ) in estimating $\mathrm{VO}_{2 \max }$ in young and healthy individuals.

\section{Materials and Methods}

\subsection{Study Sample}

Seventy-three healthy adults (37 men and 36 women; mean age: $30.8 \pm 9.3$ years, weight: $68.3 \pm 13.9 \mathrm{~kg}$, height: $168.2 \pm 10.5 \mathrm{~cm}$ ) were recruited from the university and Community Service Center at Yonsei University via advertising posters on bulletin boards from March 2013 to March 2014. Participants were screened for cardiopulmonary, orthopedic, and musculoskeletal conditions prior to testing using the Physical Activity Readiness Questionnaire and participant self-reported health history [19]. All participants provided written informed consent after explanation of experimental procedures and possible risks and benefits. The study was approved by the Institutional Review Board (IRB) of Severance Hospital, Yonsei University College of Medicine (IRB number: 4-2013-0345).

\subsection{Experimental Protocol}

The experimental protocol consisted of three clinical visits. During their first visit, anthropometric variables, such as height, weight, waist circumference, blood pressure, resting heart rate (RHR), and $\mathrm{VO}_{2 \max }$ were measured. All participants were randomly categorized into two groups (groups A and B) to avoid order effects. The participants in group A underwent the $3 \mathrm{MST}_{20}$ on their second visit and $3 \mathrm{MST}_{30}$ on their third visit; those in group $\mathrm{B}$ underwent the tests in reverse order. The third visit was scheduled 3 to 7 days after the second visit at the same hour of the day to minimize the effects circadian rhythm of the heart rate. All measurements were performed by the same investigator in a quiet and air-conditioned laboratory (temperature, $18-22^{\circ} \mathrm{C}$; humidity, $40-60 \%$ ). The participants were asked to maintain their daily living activities as usual. However, they were asked to refrain from strenuous exercise for $24 \mathrm{~h}$, drinking alcohol and caffeine for $4 \mathrm{~h}$, and eating or drinking (except water) for $2 \mathrm{~h}$ before the test. 


\subsection{Step Tests}

Step tests were conducted using the following standardized procedure. First, participants were fitted with a wearable heart rate monitor (Polar, FT1, Kempele, Finland) and then sat in a chair until a steady-state RHR was achieved (i.e., less than 5 beat-per-minute change in heart rate for one minute. Then, participants continuously stepped onto and off the box 24 times per minute for 3 min while heart rate was recorded every minute. Stepping rate was synchronized to a metronome set at 96 beats per minute and monitored throughout testing. Finally, after 3 min of stepping, participants immediately sat down in a chair while heart rate recovery was monitored for $1 \mathrm{~min}$.

\section{4. $6 M W T$}

The 6MWT was conducted according to standardized procedures provided by the American Thoracic Society [14]. It was conducted in an indoor corridor, and the course was marked by two green corns placed $30 \mathrm{~m}$ apart. Prior to the test, the examiner demonstrated the walking form and provided notifications of foul (both feet off the ground). There was no warm-up or practice beforehand. The participants rested for at least $5 \mathrm{~min}$ while sitting on a chair located near the starting position. They were asked to walk at their maximal pace to cover as much ground as possible over a 6-min period. Standardized encouragement ("keep going," "you are doing well," and "everything is going fine") was provided by the examiner during the test; other words were not allowed. The walking distance covered in meters after 6 min was recorded.

\subsection{Cardiopulmonary Exercise Test (CPET)}

CPET to measure $\mathrm{VO}_{2 \max }$ was conducted on a treadmill using a computerized cardiac stress testing system (Cardiac Science, Q-stress TM65, Waukesha, WI, USA). The participants underwent 12-lead electromyography to monitor their heart rate and cardiopulmonary stability and wore a non-rebreathing facemask (Hans Rudolph, Rudolph series 7910, Kansas, MO, USA). Oxygen consumption was continuously measured breath-by-breath using a computerized metabolic measurement system (ParvoMedics, TrueOne 2400, Sandy, UT, USA). Borg rating of perceived exertion (RPE) scale was noted every 2-min. The participants walked for a minute (speed, $1 \mathrm{mph}$; grade, $0 \%$ ) prior to the test as warm-up and familiarization to the treadmill. A well-trained investigator followed the modified Bruce protocol. $\mathrm{VO}_{2 \max }$ was considered achieved if any two of the following three criteria were met: (1) Respiratory exchange ratio of $\geq 1.1$; (2) heart rate of $>90 \%$ of age-predicted maximal heart rate; and (3) RPE of $\geq 18$. The age-predicted maximal heart rate (APMHR) was calculated using the following formula: $(208-(0.7 \times$ age $))$ [20]. All participants satisfied at least two of the $\mathrm{VO}_{2 \max }$ criteria and were then included in the analysis.

\subsection{Statistical Analysis}

The statistical significance level in all tests was set at $p<0.05$, and all analyses were performed using SPSS 25 for Windows (SPSS Inc., Chicago, IL, US). All data were presented as means \pm standard deviations. Correlation analyses were performed to assess the relationship between directly measured $\mathrm{VO}_{2 \max }$, anthropometric measurements, and outcome measures from the step tests and 6MWT. Multiple linear regressions were used to construct the $\mathrm{VO}_{2 \max }$ prediction equation to estimate $\mathrm{VO}_{2 \max }$ using variables gained from the step tests and 6MWT. In the main analysis, we used data from all participants for both model building and validation. In the sensitivity analyses, data-splitting was performed randomly to create three groups. Thereafter, model-building (two-third of the tests) and model-validation data sets (one-third of the tests) were developed. The same process was repeated two more times. Paired t-test was used to assess agreement between the estimated and actual $\mathrm{VO}_{2 \max }$. Scatter plots and Bland-Altman plots were constructed to compare the predicted and directly measured $\mathrm{VO}_{2 \mathrm{max}}$; limits of agreement were set to \pm 1.96 standard deviations from the mean. 


\section{Results}

The study population was comprised of 37 men and 36 women; participant characteristics are reported in Table 1. Among 73 participants, 64 participants completed all tests: Two step tests, $6 \mathrm{MWT}$, and $\mathrm{VO}_{2 \max }$ test. The $3 \mathrm{MST}_{20}$ was not assessed in 6 participants due to conflict of schedule and $6 \mathrm{MWT}$ was not assessed in 9 participants due to knee condition or conflict of schedule.

Table 1. Characteristics of participants.

\begin{tabular}{lccc}
\hline Variables & All & Men & Women \\
\hline$N$ & 73 & 37 & 36 \\
Age (year) & $30.8(9.3)$ & $29.4(9.5)$ & $32.3(8.9)$ \\
Weight $(\mathrm{kg})$ & $68.3(13.9)$ & $76.7(11.5)$ & $59.7(10.7)^{*}$ \\
Height $(\mathrm{cm})$ & $168.2(10.5)$ & $175.9(7.7)$ & $160.3(6.2)^{*}$ \\
BMI $\left(\mathrm{kg} / \mathrm{m}^{2}\right)$ & $24.2(3.7)$ & $24.7(2.7)$ & $23.6(4.5)$ \\
Waist circumference $(\mathrm{cm})$ & $81.8(11.1)$ & $85.4(9.1)$ & $78.0(11.8)^{*}$ \\
Resting heart rate $(\mathrm{bpm})$ & $66.2(9.4)$ & $63.2(10.2)$ & $69.2(7.6)^{*}$ \\
SBP $(\mathrm{mmHg})$ & $118.7(13.4)$ & $127.0(11.9)$ & $710.1(8.8)^{*}$ \\
DBP $(\mathrm{mmHg})$ & $75.2(9.4)$ & $77.0(9.1)$ & $73.3(9.6)$ \\
\hline
\end{tabular}

Note: BMI = body mass index; bpm = beats per minute; SBP = systolic blood pressure; DBP = diastolic blood pressure. Data are presented as means (standard deviations). ${ }^{*}$ Significant differences from men, $p<0.05$.

As shown in Table 2, heart rate increased up to $108.2 \pm 9.8 \mathrm{bpm}(57.7 \%$ APMHR) and $125.3 \pm 14.5 \mathrm{bpm}\left(67.8 \%\right.$ APMHR) at the third minute of the $3 \mathrm{MST}_{20}$ in the male and female participants, respectively; conversely, the heart rate increased up to $125.3 \pm 10.9 \mathrm{bpm}(67.0 \%$ APMHR) and $150.0 \pm 14.6 \mathrm{bpm}(81.1 \% \mathrm{APMHR})$ at the third minute of the $3 \mathrm{MST}_{30}$, respectively. The measured $\mathrm{VO}_{2 \max }$ value was $42.8 \pm 7.3$ and $31.6 \pm 6.3 \mathrm{~mL} \cdot \mathrm{kg}^{-1} \cdot \mathrm{min}^{-1}$ in the male and female participants, respectively.

Table 2. The results of maximal and submaximal tests.

\begin{tabular}{|c|c|c|c|}
\hline Heading & All & Men & Women \\
\hline $3 \mathrm{MST}_{20}$ & $N=66$ & $N=33$ & $N=33$ \\
\hline RHR (bpm) & $68.9(9.7)$ & $66.2(11.0)$ & $71.6(7.4) *$ \\
\hline HR1 (bpm) & $109.5(12.6)$ & $103.6(10.1)$ & $115.4(12.3)^{*}$ \\
\hline HR2 (bpm) & $114.1(14.6)$ & $105.8(9.5)$ & $122.6(14.3)^{*}$ \\
\hline HR3 (bpm) & $116.7(15.0)$ & $108.2(9.8)$ & $125.3(14.5)^{*}$ \\
\hline HRR30s (bpm) & $94.1(15.1)$ & $86.2(11.4)$ & $101.9(14.2)^{*}$ \\
\hline HRR1 (bpm) & $81.1(13.9)$ & $74.8(12.2)$ & $87.4(12.2)^{*}$ \\
\hline$\%$ APMHR at the third minute of exercise & $62.8(8.7)$ & $57.7(5.9)$ & $67.8(8.2) *$ \\
\hline $3 \mathrm{MST}_{30}$ & $N=73$ & $N=37$ & $N=36$ \\
\hline RHR (bpm) & $70.7(10.7)$ & $67.4(10.3)$ & $74.1(10.2) *$ \\
\hline HR1 (bpm) & $121.6(13.2)$ & $114.3(8.6)$ & $129.1(12.9)^{*}$ \\
\hline HR2 (bpm) & $132.9(15.6)$ & $122.3(10.4)$ & $143.8(12.2)^{*}$ \\
\hline HR3 (bpm) & $137.5(17.8)$ & $125.3(10.9)$ & $150.0(14.6)^{*}$ \\
\hline HRR30s (bpm) & $112.9(20.5)$ & $101.9(14.9)$ & $124.3(19.4)^{*}$ \\
\hline HRR1 (bpm) & $96.5(20.4)$ & $86.1(15.0)$ & $107.1(19.8)^{*}$ \\
\hline$\% A P M H R$ at the third minute of exercise & $73.9(10.8)$ & $67.0(7.2)$ & $81.1(9.0) *$ \\
\hline Six-minute walk test & $N=64$ & $N=32$ & $N=32$ \\
\hline Distance (m) & $715(94.9)$ & $762.7(97.2)$ & $667.4(64.6) *$ \\
\hline Cardiopulmonary exercise test & $N=73$ & $N=37$ & $N=36$ \\
\hline RHR (bpm) & $19.01(1.1)$ & $19.0(1.0)$ & $19.0(1.2)$ \\
\hline HR at end of the test (bpm) & $188.8(10.3)$ & $191.6(8.7)$ & $185.9(11.2)^{*}$ \\
\hline \%APMHR & $101.3(5.5)$ & $102.3(5.0)$ & $100.3(5.8)$ \\
\hline Cessation stage (range) & $5.2(3-6)$ & $5.7(5-6)$ & $4.6(3-6)$ \\
\hline $\mathrm{VO}_{2 \max }\left(\mathrm{mL} \cdot \mathrm{kg}^{-1} \cdot \mathrm{min}^{-1}\right)$ & $37.3(8.8)$ & $42.8(7.3)$ & $31.6(6.3) *$ \\
\hline
\end{tabular}

Note: $3 \mathrm{MST}_{20}=3$-min step test using 20.3-cm step box height; RHR = resting heart rate; bpm = beats per minute; $\mathrm{HR} 1$ = heart rate at $1 \mathrm{~min} ; \mathrm{HR} 2=$ heart rate at $2 \mathrm{~min} ; \mathrm{HR} 3=$ heart rate at $3 \mathrm{~min} ; \mathrm{HRR} 30 \mathrm{~s}=$ heart rate recovery at $30 \mathrm{~s}$ after cessation; HRR1 = heart rate recovery at $1 \mathrm{~min}$ after cessation; APMHR = age-predicted maximal heart rate; $3 \mathrm{MST}_{30}=3$-min step test using 30 -cm step box height; $\mathrm{HR}=$ heart rate; $\mathrm{VO}_{2 \max }=$ maximal oxygen consumption. Numerical variables are presented as means (standard deviations) and categorical variables as means (ranges).

* Significant differences from the male participants, $p<0.05$. 
We performed correlation analyses to determine the association between step test heart rate recovery measures and directly measured $\mathrm{VO}_{2 \max }$ (Supplementary Table S1). In both the $3 \mathrm{MST}_{20}$ and $3 \mathrm{MST}_{30}$, heart rate recovery at $30 \mathrm{~s}$ (HRR30s) showed the strongest correlation with the measured $\mathrm{VO}_{2 \max }$ (Supplementary Table S1); therefore, it was used for the $\mathrm{VO}_{2 \max }$ estimation model. The HRR30s attributed to the $32.8 \%$ of $\mathrm{VO}_{2 \max }$ and $45.8 \%$ of $\mathrm{VO}_{2 \max }$ for the $3 \mathrm{MST}_{20}$ and $3 \mathrm{MST}_{30}$, respectively. When the HRR30s was complemented with sex, age, and weight, the model showed $73.4 \%$ and $72.2 \%$ of $\mathrm{VO}_{2 \mathrm{max}}$, for $3 \mathrm{MST}_{20}$ and $3 \mathrm{MST}_{30}$ respectively. The distance walked during the $6 \mathrm{MWT}$ attributed to $44.1 \%$ of $\mathrm{VO}_{2 \max }$, which increased to $74.4 \%$ when sex, age, and weight were added to the model (Table 3).

Table 3. Prediction equation model development using the different submaximal tests.

\begin{tabular}{|c|c|c|c|c|c|c|c|c|c|c|}
\hline \multirow[b]{2}{*}{ Tests } & \multirow[b]{2}{*}{ Models } & \multirow{2}{*}{$\begin{array}{c}\text { Adjusted } \\
\qquad \mathbf{R}^{2}\end{array}$} & \multirow[b]{2}{*}{ SEE } & \multirow[b]{2}{*}{$\Delta \mathrm{F}$} & \multicolumn{6}{|c|}{ Coefficient Estimates } \\
\hline & & & & & Intercept & HRR30s & Sex & Age & Weight & $\begin{array}{c}\text { Distance } \\
\text { Walked } \cdot 10^{-1}\end{array}$ \\
\hline \multirow{4}{*}{$3 \mathrm{MST}_{20}$} & Model 1 & 0.328 & 7.4 & 36.7 * & $69.9 *$ & $-12.0^{*}$ & & & & \\
\hline & Model 2 & 0.503 & 6.4 & 33.9 * & $68.5 *$ & $-0.2 *$ & $-9.0 *$ & & & \\
\hline & Model 3 & 0.713 & 4.9 & 54.7 * & $76.4 *$ & $-0.1 *$ & $-8.0 *$ & $-0.5^{*}$ & & \\
\hline & Model 4 & 0.734 & 4.7 & $45.9^{*}$ & $86.0 *$ & $-0.1 *$ & $-10.9^{*}$ & $-0.4^{*}$ & $-0.1^{*}$ & \\
\hline \multirow{4}{*}{$3 \mathrm{MST}_{30}$} & Model 1 & 0.458 & 6.5 & 61.9 * & $70.5 *$ & $-0.3 *$ & & & & \\
\hline & Model 2 & 0.555 & 5.9 & 45.9 * & $70.3 *$ & -0.2 * & $-6.7^{*}$ & & & \\
\hline & Model 3 & 0.699 & 4.8 & $56.8 *$ & $73.7 *$ & $-0.1 *$ & $-7.6 *$ & -0.4 * & & \\
\hline & Model 4 & 0.722 & 4.7 & $47.7^{*}$ & $84.5^{*}$ & $-0.1 *$ & $-10.2 *$ & $-0.4^{*}$ & $-0.1^{*}$ & \\
\hline \multirow{4}{*}{$6 \mathrm{MWT}$} & Model 1 & 0.441 & 6.8 & 50.8 * & -9.09 & & & & & $0.6^{*}$ \\
\hline & Model 2 & 0.576 & 5.9 & 43.8 * & 17.6 * & & $-7.8 *$ & & & 0.4 * \\
\hline & Model 3 & 0.699 & 5.0 & 49.7 * & 45.7 * & & $-8.2 *$ & $-0.4^{*}$ & & $0.2 *$ \\
\hline & Model 4 & 0.744 & 4.6 & $46.8^{*}$ & $61.1 *$ & & $-11.2 *$ & -0.4 * & $-0.2 *$ & $0.2 *$ \\
\hline
\end{tabular}

Note: $\mathrm{SEE}=$ standard error of estimate; HRR30s $=$ heart rate recovery at $30 \mathrm{~s} ; 3 \mathrm{MST}_{20}=3$-min step test using 20-cm step height; $3 \mathrm{MST}_{30}=3$-min step test using 30 -cm step height; $6 \mathrm{MWT}=6$-min walk test. ${ }^{*} p<0.05$.

Thereafter, we used multiple linear regressions to develop the prediction equation for $\mathrm{VO}_{2 \max }$ estimation for each of the submaximal tests.

- 3MST $_{20}: V O_{2 \max }=86.0-10.9 \times \operatorname{sex}($ male $=1$, female $=2)-0.4 \times$ age $-0.1 \times$ weight $-0.1 \times$ HRR30s

- 3MST $_{30}: V O_{2 \max }=84.5-10.2 \times \operatorname{sex}($ male $=1$, female $=2)-0.4 \times$ age $-0.1 \times$ weight $0.1 \times H R R 30 s$

- 6MWT: $V O_{2 \max }=61.1-11.1 \times \operatorname{sex}($ male $=1$, female $=2)-0.4 \times$ age $-0.2 \times$ weight $0.2 \times\left(\right.$ distance walked $\left.\cdot 10^{-1}\right)$

Using these equations, we calculated $\mathrm{VO}_{2 \max }$ for each participant. The estimated $\mathrm{VO}_{2 \max }$ values were $37.0 \pm 7.9,37.3 \pm 7.6$, and $36.9 \pm 7.9 \mathrm{~mL} \cdot \mathrm{kg}^{-1} \cdot \mathrm{min}^{-1}$ derived from the $3 \mathrm{MST}_{20}, 3 \mathrm{MST}_{30}$, and $6 \mathrm{MWT}$, respectively; the $\mathrm{R}^{2}$ values between the estimated and measured $\mathrm{VO}_{2 \max }$ were $0.734,0.722$, and 0.744 , respectively (Figure 1).
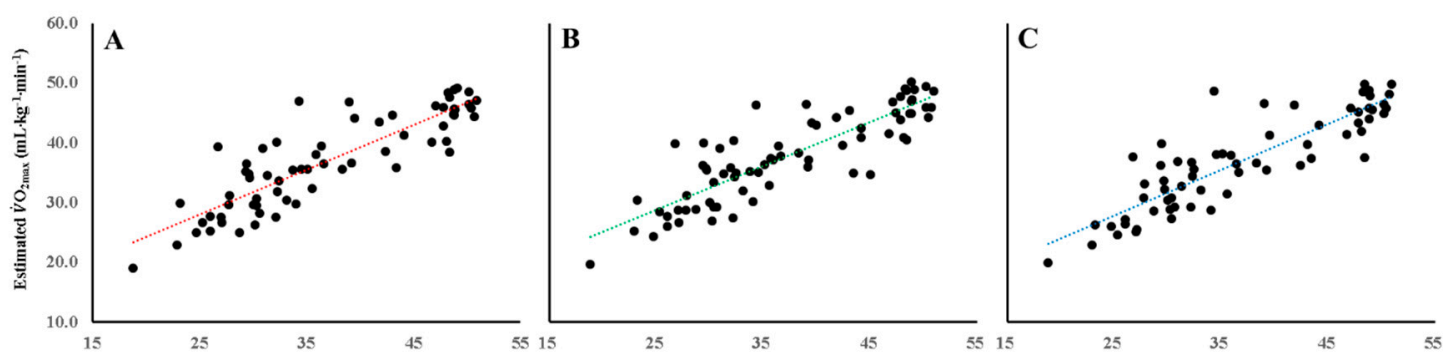

Measured $\dot{V O}_{2 \max }\left(\mathrm{mL}^{2} \mathrm{~kg}^{-1} \cdot \mathrm{min}^{-1}\right)$

Figure 1. Correlations between measured $\mathrm{VO}_{2 \max }$ and model-predicted $\mathrm{VO}_{2 \max }$ using $2 \mathrm{MST}_{20}(\mathrm{r}=0.866$ $p<0.05)(\mathbf{A}), 3 \mathrm{MST}_{30}(\mathrm{r}=0.859, p<0.05)(\mathbf{B})$, and $6 \mathrm{MWT}(\mathrm{r}=0.872, p<0.05)(\mathbf{C})$. 
There were no significant differences between the predicted and directly measured $\mathrm{VO}_{2 \max }$ values. Furthermore, the Bland-Altman plot analysis showed no notable difference in the agreement between the estimated and measured $\mathrm{VO}_{2 \max }$ according to sex in all three submaximal tests (Figure 2).
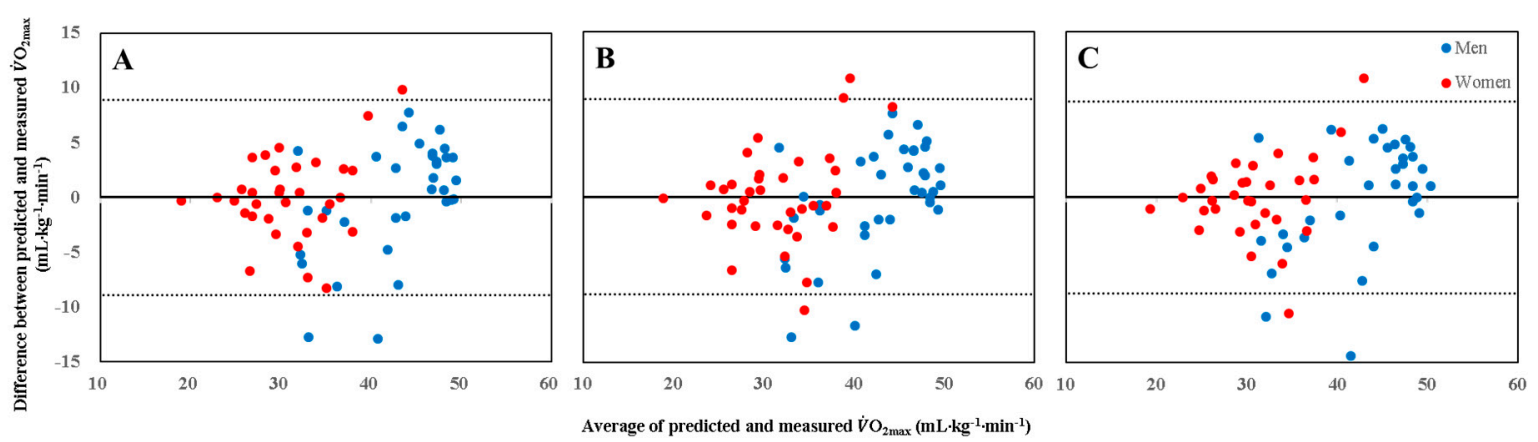

Figure 2. Bland-Altman plots show agreement of mean differences between measured $\mathrm{VO}_{2 \max }$ and estimated $\mathrm{VO}_{2 \text { max }}$ obtained from $3 \mathrm{MWT}_{20}(\mathbf{A}), 3 \mathrm{MWT}_{30}(\mathbf{B})$, and $6 \mathrm{MWT}(\mathbf{C})$. Solid lines represent mean differences between measured $\mathrm{VO}_{2 \max }$ and estimated $\mathrm{VO}_{2 \text { max. }}$. Upper and lower dot lines represent $95 \%$ limits of agreement (mean difference \pm SD of differences).

Since data from same participants were used for building the prediction equation and validation, we performed further sensitivity analyses using three-fold cross validation methods; the participants in two groups were used to develop the prediction equation and participants in one group to validate the estimated results. The same methods were repeated three times (Supplementary Tables S2-S4 and Figures S1-S3), and the results were similar to those in our main analyses. The adjusted $\mathrm{R}^{2}$ value between the estimated $\mathrm{VO}_{2 \max }$ using the three different estimation equations from the $3 \mathrm{MST}_{20}$ and measured $\mathrm{VO}_{2 \max }$ ranged from 0.702 to 0.773 . The adjusted $\mathrm{R}^{2}$ value between the estimated $\mathrm{VO}_{2 \max }$ using the three different estimation equations from the $3 \mathrm{MST}_{30}$ and measured $\mathrm{VO}_{2 \text { max }}$ ranged from 0.661 to 0.803 . The adjusted $\mathrm{R}^{2}$ value between the estimated $\mathrm{VO}_{2 \max }$ using the three different estimation equations from the $6 \mathrm{MWT}$ and measured $\mathrm{VO}_{2 \max }$ ranged from 0.700 to 0.820 .

\section{Discussion}

As cardiopulmonary fitness is often used to predict health status, mortality and the prevalence or incidence of diseases [1], safe, convenient, and valid measurement to assess such is of great interest for epidemiological studies. In our study, we have demonstrated that two steps tests $\left(3 \mathrm{MST}_{20}, 3 \mathrm{MST}_{30}\right.$ ) as well as the 6MWT were valid method in estimating $\mathrm{VO}_{2 \max }$ in relatively young and healthy population. We also confirmed that the $3 \mathrm{MST}_{20}$ was not inferior to the $3 \mathrm{MST}_{30}$ in estimating $\mathrm{VO}_{2 \max }$, suggesting that a lower step height is as good as a higher step height.

Owing to its convenience, many step test protocols have been developed and validated $[2,7,10,21]$. In incremental step tests, participants' ability to perform incremental work and physiological response to the work are usually observed [4]; the step height, test duration, stepping rate, RPE, and heart rate response during the test and/or recovery are also considered. However, these tests have several assumptions: Participants' will to complete the tests, validity and reliability of the RPE, and validity of the APMHR. Thus, the step test protocol, which eliminates these assumptions from the test, might be more valid. In this context, we validated relatively short $(3 \mathrm{~min}$ ) and single-stage (fixed step height and stepping rate) step tests. As the step height significantly influences the participants' ability to complete the test owing to possible presence of local muscle fatigue, lower extremity joint pain, and physical function impairment, we tested two different 3-min step test protocols at 20.3 and $30 \mathrm{~cm}$, conducted on separate days in random order. As expected, the heart rate increase was higher during the step test using higher step height was used. However, we found no difference in the validity of the two different protocols in estimating $\mathrm{VO}_{2 \max }$, suggesting that the $3 \mathrm{MST}_{20}$ is as good as the $3 \mathrm{MST}_{30}$ in estimating $\mathrm{VO}_{2 \max }$. 
Demonstrating the $3 \mathrm{MST}_{20}$ is not inferior to the $3 \mathrm{MST}_{30}$ is of interest for several reasons. Owing to the lower step height, the $3 \mathrm{MST}_{20}$ is more feasible for those with lower extremity joint problems, obese and unfit individuals. Previously, Bohannon et al. [11] compared between the 6MWT and YMCA step test and found relatively low completion rates in the latter; 51 out of 189 participants did not complete the test. The average age of those who did not complete the step test was $70.4 \pm 14.2$ years, while that of those who completed the test was $39.9 \pm 19.4$ years. Similarly, Beutner et al. [2] also reported that $17 \%$ of their participants did not complete 3 min YMCA step test; participants who were not able to complete the 3 min tests were older (53.2 vs. 69.3 years) and more obese (BMI: 24.5 vs. $29.5 \mathrm{~kg} / \mathrm{m}^{2}$ ). As most study populations had a wide range of age and health status, we can speculate that the $3 \mathrm{MST}_{20}$, which uses a lower step height, would be more feasible. Furthermore, our study showed that the $3 \mathrm{MST}_{20}$ and $3 \mathrm{MST}_{30}$ increased the heart rate up to $62.8 \%$ and $73.9 \%$ of the APMHR, respectively. In the female participants, the heart rate increased up to $81.1 \%$ of their APMHR during the $3 \mathrm{MST}_{30}$, which suggests that the $3 \mathrm{MST}_{30}$ is a moderate to vigorous intensity exercise. Therefore, the $3 \mathrm{MST}_{20}$ can be more suitable for those with potential risks of cardiac events. However, we did not validate the $3 \mathrm{MST}_{20}$ for prediction of $\mathrm{VO}_{2 \max }$ in clinical populations in our study.

One of the strengths of single-stage step tests is the lack of influence by the will of participants, especially with shorter durations (e.g., $3 \mathrm{~min}$ ) and lower step heights. The heart rate only increased up to $60-70 \%$ of the APMHR during the $3 \mathrm{MST}_{20}$, which suggests relatively low physical stress to the participants during the test. As participants cannot manipulate their own heart rate, the $3 \mathrm{MST}_{20}$ using heart rate recovery after submaximal exercise may yield objective results. In this context, single-stage step tests with lower step heights rather than multi-stage step tests may be more reliable. For example, the Chester step test lasts up to $10 \mathrm{~min}$ and uses a 30-cm step box with an incremental stepping rate [4,7]; it ends when participants (1) cannot continue the test, (2) have reached $80 \%$ of their APMHR, and (3) have reached $10 \mathrm{~min}$ into the test. Therefore, single-stage 3-min step tests with low step heights, such as the Tecumseh step test, might be a safe, objective, inexpensive, convenient, and valid test to assess the level of aerobic fitness.

Although both the $3 \mathrm{MST}_{20}$ and $3 \mathrm{MST}_{30}$ were valid in estimating $\mathrm{VO}_{2 \max }$, it is important to note that the prediction equations also included other variables, such as age, sex, and body weight. When only HRR30s during the $3 \mathrm{MST}_{20}$ and $3 \mathrm{MST}_{30}$ was used as a predicting variable, a stronger correlation was observed between the HRR30s and actual $\mathrm{VO}_{2 \max }$ during $3 \mathrm{MST}_{30}$. When the prediction equation was supplemented with age, sex, and body weight, the prediction equation developed for both step tests explained the actual $\mathrm{VO}_{2 \max }$ to an equal extent.

Our study also validated the $6 \mathrm{MWT}$ in estimating $\mathrm{VO}_{2 \max }$. In 1968, Cooper developed and validated a 12-min run test to estimate $\mathrm{VO}_{2 \max }$ in male US Air Force officers and personnel [22]. Later, McGavin et al. [23] modified this test as a 12-min walk test to assess lung function among patients with chronic bronchitis, which later became the 6-min walk test (3MWT) [24]. Since then, the 6MWT has been validated in various clinical populations [25], including those with cancer [26,27], although a recent study did not recommend the use of the $6 \mathrm{MWT}$ to assess $\mathrm{VO}_{2 \max }$ in patients with cancer [28]. In the current study, the $6 \mathrm{MWT}$ was also valid in estimating $\mathrm{VO}_{2 \max }$ of the relatively young and healthy participants. The $\mathrm{VO}_{2 \max }$ estimated using the prediction equation derived from the 6MWT agrees with the actual $\mathrm{VO}_{2 \max }\left(36.8\right.$ vs. $\left.36.9 \mathrm{~mL} \cdot \mathrm{kg}^{-1} \cdot \mathrm{min}^{-1}\right)$. When the model-building and model-validation sets were randomly assigned, the estimated $\mathrm{VO}_{2 \max }$ in the $6 \mathrm{MWT}$ was still valid, with a correlation coefficient between 0.771 and 0.910 .

Our study has strengths and limitations. One of the strengths of our study is that three different submaximal tests were validated in estimating $\mathrm{VO}_{2 \max }$ against the measured $\mathrm{VO}_{2 \max }$ among the same participants. Furthermore, we provided evidence that the use of short-duration step tests using a low step box $(20.3 \mathrm{~cm})$, which elicited a significantly lower heart rate than the step test using a high step box, is equally valid in estimating $\mathrm{VO}_{2 \max }$ in young and healthy populations. Traditionally, a good step test encourages higher steps, higher stepping rates, and longer durations; which mimics the maximal test. However, we clearly demonstrated that submaximal tests using heart rate or distance walked in 
6 min were sufficient in estimating $\mathrm{VO}_{2 \max }$ in healthy populations. Conversely, our study limitation is the relatively small convenience sample from a university and community service center in Korea; therefore, the participants were generally young and healthy Koreans. Thus, caution should be taken in applying our results to the elderly, clinical populations, and other ethnicities. Another limitation is the use of the same sample for model building and validation in estimating $\mathrm{VO}_{2 \max }$. To overcome this limitation, we performed sensitivity analyses by randomly splitting our sample into three groups and cross-validating them. These analyses showed that our submaximal tests were all still valid in estimating $\mathrm{VO}_{2 \max }$. Further research with a broader range of populations will, however, be required to enhance the validity of the prediction equations of the tests.

\section{Conclusions}

We validated the 3-min step test using two different step box heights and the 6MWT in a relatively young and healthy Asian population; these tests can be used to provide valid estimates of $\mathrm{VO}_{2 \max }$ in epidemiological studies.

Supplementary Materials: The following are available online at http://www.mdpi.com/1660-4601/16/23/4858/s1, Figure S1: Correlations between the measured VO2max and model-predicted VO2max in the 3-min step test using a 20-cm step height. The prediction equation model was developed using randomly selected two-third of the participants, and their value was compared with that of the non-selected one-third of the participants. The same methods were repeated three times, Figure S2: Correlations between the measured VO2max and model-predicted VO2max in the 3-min step test using a 30-cm step height. The prediction equation model was developed using randomly selected two-third of the participants, and their value was compared with that of the non-selected one-third of the participants. The same methods were repeated three times, Figure S3: Correlations between the measured $\mathrm{VO} 2 \mathrm{max}$ and model-predicted $\mathrm{VO} 2 \mathrm{max}$ in the 6-min walk test. The prediction equation model was developed using randomly selected two-third of the participants, and their value was compared with that of the non-selected one-third of the participants. The same methods were repeated three times, Table S1: Correlation between the measured VO2max, anthropometrics, and variables acquired from the submaximal tests, Table S2: Correlations between the measured VO2max and model-predicted VO2max in the 3-min step test using a 20-cm step height. The prediction equation model was developed using randomly selected two-third of the participants, and their value was compared with that of the non-selected one-third of the participants. The same methods were repeated three times, Table S3: Correlations between the measured VO2max and model-predicted VO2max in the 3-min step test using a $30-\mathrm{cm}$ step height. The prediction equation model was developed using randomly selected two-third of the participants, and their value was compared with that of the non-selected one-third of the participants. The same methods were repeated three times, Table S4: Correlations between the measured VO2max and model-predicted VO2max in the 6-min walk test. The prediction equation model was developed using randomly selected two-third of the participants, and their value was compared with that of the non-selected one-third of the participants. The same methods were repeated three times.

Author Contributions: Conceptualization, J.Y.J. and S.H.H.; Methodology, J.Y.J. and S.H.H.; formal analysis, S.H.H.; investigation, S.H.H., H.I.Y., and D.-I.K.; data curation, S.H.H.; writing—original draft preparation, S.H.H.; writing-review and editing, J.Y.J., T.I.G., S.B.

Funding: This study was supported by a grant from the National R\&D Program for Cancer Control, Ministry of Health \& Welfare, Republic of Korea (HA16C0012).

Conflicts of Interest: The authors declare no conflict of interest.

\section{References}

1. Ross, R.; Blair, S.N.; Arena, R.; Church, T.S.; Despres, J.P.; Franklin, B.A.; Haskell, W.L.; Kaminsky, L.A.; Levine, B.D.; Lavie, C.J.; et al. Importance of Assessing Cardiorespiratory Fitness in Clinical Practice: A Case for Fitness as a Clinical Vital Sign: A Scientific Statement From the American Heart Association. Circulation 2016, 134, e653-e699. [CrossRef] [PubMed]

2. Beutner, F.; Ubrich, R.; Zachariae, S.; Engel, C.; Sandri, M.; Teren, A.; Gielen, S. Validation of a brief step-test protocol for estimation of peak oxygen uptake. Eur. J. Prev. Cardiol. 2015, 22, 503-512. [CrossRef] [PubMed]

3. Patton, J.F.; Vogel, J.A.; Mello, R.P. Evaluation of a maximal predictive cycle ergometer test of aerobic power. Eur. J. Appl. Physiol. Occup. Physiol. 1982, 49, 131-140. [CrossRef] [PubMed]

4. Bennett, H.; Parfitt, G.; Davison, K.; Eston, R. Validity of Submaximal Step Tests to Estimate Maximal Oxygen Uptake in Healthy Adults. Sports Med. 2016, 46, 737-750. [CrossRef] [PubMed] 
5. George, J.D.; Vehrs, P.R.; Allsen, P.E.; Fellingham, G.W.; Fisher, A.G. Development of a submaximal treadmill jogging test for fit college-aged individuals. Med. Sci. Sports Exerc. 1993, 25, 643-647. [CrossRef]

6. Hughes, A.D.; Chaturvedi, N. Estimation of Maximal Oxygen Consumption and Heart Rate Recovery Using the Tecumseh Sub-Maximal Step Test and their Relationship to Cardiovascular Risk Factors. Artery Res. 2017, 18, 29-35. [CrossRef]

7. Buckley, J.P.; Sim, J.; Eston, R.G.; Hession, R.; Fox, R. Reliability and validity of measures taken during the Chester step test to predict aerobic power and to prescribe aerobic exercise. Br. J. Sports Med. 2004, 38, 197-205. [CrossRef]

8. JETTÉ, M. A calculator to predict maximal oxygen consumption for use with the Canadian Home Fitness Test. Can. J. Public Health 1977, 68, 195-198.

9. Shephard, R.J. The current status of the Canadian home fitness test. Br. J. Sports Med. 1980, 14, 114-125. [CrossRef]

10. Shephard, R.J.; Bailey, D.A.; Mirwald, R.L. Development of the Canadian Home Fitness Test. Can. Med. Assoc. J. 1976, 114, 675-679.

11. Bohannon, R.W.; Bubela, D.J.; Wang, Y.-C.; Magasi, S.S.; Gershon, R.C. Six-minute walk test versus three-minute step test for measuring functional endurance (Alternative Measures of Functional Endurance). J. Strength Cond. Res./Natl. Strength Cond. Assoc. 2015, 29, 3240. [CrossRef] [PubMed]

12. Enright, P.L.; McBurnie, M.A.; Bittner, V.; Tracy, R.P.; McNamara, R.; Arnold, A.; Newman, A.B.; Cardiovascular Health, S. The 6-min walk test: A quick measure of functional status in elderly adults. Chest 2003, 123, 387-398. [CrossRef] [PubMed]

13. du Bois, R.M.; Weycker, D.; Albera, C.; Bradford, W.Z.; Costabel, U.; Kartashov, A.; Lancaster, L.; Noble, P.W.; Sahn, S.A.; Szwarcberg, J.; et al. Six-minute-walk test in idiopathic pulmonary fibrosis: Test validation and minimal clinically important difference. Am. J. Respir. Crit. Care Med. 2011, 183, 1231-1237. [CrossRef] [PubMed]

14. ATS Committee on Proficiency Standards for Clinical Pulmonary Function Laboratories. ATS statement: Guidelines for the six-minute walk test. Am. J. Respir. Crit. Care Med. 2002, 166, 111-117. [CrossRef]

15. Aquino, E.S.; Mourao, F.A.; Souza, R.K.; Glicerio, B.M.; Coelho, C.C. Comparative analysis of the six-minute walk test in healthy children and adolescents. Rev. Bras. Fisioter. 2010, 14, 75-80. [CrossRef] [PubMed]

16. Morinder, G.; Mattsson, E.; Sollander, C.; Marcus, C.; Larsson, U.E. Six-minute walk test in obese children and adolescents: Reproducibility and validity. Physiother. Res. Int. 2009, 14, 91-104. [CrossRef] [PubMed]

17. Rauchenzauner, M.; Schneider, J.; Colleselli, V.; Ruepp, M.; Cortina, G.; Hogler, W.; Neururer, S.; Geiger, R. Comparing modalities of conducting the six-minute walk test in healthy children and adolescents. Minerva Pediatr. 2019, 71, 229-234. [CrossRef]

18. Takken, T. Six-minute walk test is a poor predictor of maximum oxygen uptake in children. Acta Paediatr. 2010, 99, 958. [CrossRef]

19. Thomas, S.; Reading, J.; Shephard, R.J. Revision of the Physical Activity Readiness Questionnaire (PAR-Q). Can. J. Sport Sci. 1992, 17, 338-345.

20. Tanaka, H.; Monahan, K.D.; Seals, D.R. Age-predicted maximal heart rate revisited. J. Am. Coll. Cardiol. 2001, 37, 153-156. [CrossRef]

21. Kasch, F.W.; Phillips, W.H.; Ross, W.D.; Carter, J.E.; Boyer, J.L. A comparison of maximal oxygen uptake by treadmill and step-test procedures. J. Appl. Physiol. 1966, 21, 1387-1388. [CrossRef] [PubMed]

22. Cooper, K.H. A means of assessing maximal oxygen intake. Correlation between field and treadmill testing. JAMA 1968, 203, 201-204. [CrossRef] [PubMed]

23. McGavin, C.; Gupta, S.; McHardy, G. Twelve-minute walking test for assessing disability in chronic bronchitis. Br. Med. J. 1976, 1, 822-823. [CrossRef] [PubMed]

24. Butland, R.; Pang, J.; Gross, E.; Woodcock, A.; Geddes, D. Two-, six-, and 12-min walking tests in respiratory disease. Br. Med. J. (Clin. Res. Ed.) 1982, 284, 1607. [CrossRef]

25. Ross, R.M.; Murthy, J.N.; Wollak, I.D.; Jackson, A.S. The six minute walk test accurately estimates mean peak oxygen uptake. BMC Pulm. Med. 2010, 10, 31. [CrossRef]

26. Cavalheri, V.; Jenkins, S.; Cecins, N.; Gain, K.; Hill, K. Comparison of the six-minute walk test with a cycle-based cardiopulmonary exercise test in people following curative intent treatment for non-small cell lung cancer. Chronic Respir. Dis. 2016, 13, 118-127. [CrossRef] 
27. Schmidt, K.; Vogt, L.; Thiel, C.; Jäger, E.; Banzer, W. Validity of the six-minute walk test in cancer patients. Int. J. Sports Med. 2013, 34, 631-636. [CrossRef]

28. Schumacher, A.N.; Shackelford, D.Y.; Brown, J.M.; Hayward, R. Validation of the 6-min Walk Test for Predicting Peak VO2 in Cancer Survivors. Med. Sci. Sports Exerc. 2019, 51, 271-277. [CrossRef]

(C) 2019 by the authors. Licensee MDPI, Basel, Switzerland. This article is an open access article distributed under the terms and conditions of the Creative Commons Attribution (CC BY) license (http://creativecommons.org/licenses/by/4.0/). 